\title{
Comparison of sliding window and field-in-field techniques for tangential whole breast irradiation using the Halcyon and Synergy Agility systems
}

Anne Richter * (1), Sonja Wegener, Kathrin Breuer, Gary Razinskas, Stefan Weick, Florian Exner, Klaus Bratengeier, Michael Flentje, Otto Sauer and Bülent Polat

\begin{abstract}
Background: To implement a tangential treatment technique for whole breast irradiation using the Varian Halcyon and to compare it with Elekta Synergy Agility plans.

Methods: For 20 patients two comparable treatment plans with respect to dose coverage and normal tissue sparing were generated. Tangential field-in-field treatment plans (Pinnacle/Synergy) were replanned using the sliding window technique (Eclipse/Halcyon). Plan specific QA was performed using the portal Dosimetry and the ArcCHECK phantom. Imaging and treatment dose were evaluated for treatment delivery on both systems using a modified CIRS Phantom.

Results: The mean number of monitor units for a fraction dose of 2.67 Gy was 515 MUs and 260 MUs for Halcyon and Synergy Agility plans, respectively. The homogeneity index and dose coverage were similar for both treatment units. The plan specific QA showed good agreement between measured and calculated plans. All Halcyon plans passed portal dosimetry QA (3\%/2 mm) with 100\% points passing and ArcCheck QA (3\%/2 mm) with 99.5\%. Measurement of the cumulated treatment and imaging dose with the CIRS phantom resulted in lower dose to the contralateral breast for the Halcyon plans.
\end{abstract}

Conclusions: For the Varian Halcyon a plan quality similar to the Elekta Synergy device was achieved. For the Halcyon plans the dose contribution from the treatment fields to the contralateral breast was even lower due to less interleaf transmission of the Halcyon MLC and a lower contribution of scattered dose from the collimator system.

Keywords: Whole breast irradiation, Halcyon, IGRT, Dose to OARs

\section{Background}

Breast cancer is one of the most common malignant tumors in women. Standard treatment for early-stage breast cancer is breast-conserving surgery followed by adjuvant whole breast irradiation [1].

\footnotetext{
*Correspondence: richter_a3@ukw.de
}

Department of Radiation Oncology, University of Wuerzburg, Wuerzburg, Germany
The sparing of the organs at risk is important as the long term survival probability for diagnosed stage I and II patients treated with radiation after breast-conserving therapy is high $[2,3]$. Different treatment modalities are available for radiation delivery like three dimensional conformal therapy (3D-CRT) $[4,5]$, tomotherapy [6-9], step-and-shoot or dynamic sliding window intensitymodulated radiation therapy (IMRT) and volumetric modulated arc therapy (VMAT) [9-11]. original author(s) and the source, provide a link to the Creative Commons licence, and indicate if changes were made. The images or other third party material in this article are included in the article's Creative Commons licence, unless indicated otherwise in a credit line to the material. If material is not included in the article's Creative Commons licence and your intended use is not permitted by statutory regulation or exceeds the permitted use, you will need to obtain permission directly from the copyright holder. To view a copy of this licence, visit http://creativecommons.org/licenses/by/4.0/. The Creative Commons Public Domain Dedication waiver (http://creativeco mmons.org/publicdomain/zero/1.0/) applies to the data made available in this article, unless otherwise stated in a credit line to the data. 
The Varian Halcyon linac offers 6 MV flattening-filter-free (FFF) photon beam energy with a dual-layer multi-leaf collimator (MLC). Treatment on the Halcyon requires daily image-guidance because there are no light field, optical distance indicator or lasers at the treatment isocenter available.

The first aim of this work was to implement a treatment technique suitable for tangential whole breast irradiation using the Halcyon - with similar plan quality compared to Synergy Agility. Second aim was to investigate the dosimetric effect of daily imaging on the Halcyon unit for the contralateral breast. A comparison of the Halcyon and Synergy Agility plans in terms of imaging dose and deliverability was performed by QA measurements.

\section{Methods}

\section{Patient characteristics}

All patient characteristics are listed in Table 1. The patients were treated via tangential whole breast irradiation without supraclavicular or internal mammary lymph nodes. The size of the planning target volumes ranged from $351 \mathrm{~cm}^{3}$ to $1439 \mathrm{~cm}^{3}$ with a mean target volume of $814 \mathrm{~cm}^{3}$. The cohort consisted of two subgroups. The first ten patients were treated with Elekta Synergy Agility (Elekta Medical Systems, Crawley, UK). Treatment plans with tangential beams were re-planned for Halcyon to compare the plan quality and dose to OARs retrospectively. The second subgroup of 10 patients was scheduled for whole breast irradiation on the Varian Halcyon system (Varian Medical Systems, Palo Alto, CA, USA, version 2.0) while treatment plans for Halcyon and Synergy Agility were created. Finally, two comparable plans were available for all patients.

\section{Treatment planning}

Patients were positioned in supine position with both arms raised. CT imaging and treatment were performed in free breathing. The dose prescription was $40 \mathrm{~Gy}$ in 15 fractions followed by a boost of 10 Gy in 5 fractions (mean dose). PTV was delineated closed to the surface. Dose homogeneity was maintained by the standard deviation (SD) in PTV-5 mm of less than 3\%. PTV-0.5 mm corresponds to the CTV as defined in the ESTRO Guideline $[12,13]$.

Pinnacle (Philips Radiation Oncology Systems, Fitchburg, WI, version 16.2) was used for treatment planning

Table 1 Patient and treatment characteristics

\begin{tabular}{|c|c|c|c|c|c|c|c|c|c|}
\hline \multirow[t]{2}{*}{ Treatment machine } & \multirow[t]{2}{*}{ Patient } & \multirow[t]{2}{*}{ Tumor site } & \multirow[t]{2}{*}{ PTV in $\mathrm{cm}^{3}$} & \multicolumn{2}{|l|}{ Energy } & \multicolumn{2}{|l|}{ Fields } & \multicolumn{2}{|c|}{ Monitor Units } \\
\hline & & & & Synergy & Halcyon & Synergy & Halcyon & Synergy & Halcyon \\
\hline \multirow[t]{10}{*}{ Synergy Agility } & 1 & Left & 690 & $6 \& 10 M V$ & $6 \mathrm{MV} \mathrm{FFF}$ & 4 & 2 & 245 & 446 \\
\hline & 2 & Left & 1163 & $6 \mathrm{MV}$ & $6 \mathrm{MV} \mathrm{FFF}$ & 4 & 2 & 268 & 499 \\
\hline & 3 & Left & 832 & $6 \mathrm{MV}$ & $6 \mathrm{MV} \mathrm{FFF}$ & 4 & 2 & 261 & 502 \\
\hline & 4 & Left & 946 & $6 \mathrm{MV}$ & 6MV FFF & 4 & 2 & 263 & 440 \\
\hline & 5 & Left & 435 & $6 \mathrm{MV}$ & $6 \mathrm{MV} \mathrm{FFF}$ & 4 & 2 & 253 & 436 \\
\hline & 6 & Right & 790 & $6 \mathrm{MV}$ & $6 \mathrm{MV} \mathrm{FFF}$ & 4 & 2 & 256 & 470 \\
\hline & 7 & Right & 509 & $6 \& 10 M V$ & 6MV FFF & 5 & 2 & 254 & 470 \\
\hline & 8 & Right & 934 & $6 \mathrm{MV}$ & 6MV FFF & 4 & 2 & 269 & 494 \\
\hline & 9 & Right & 351 & $6 \& 10 M V$ & 6MV FFF & 5 & 2 & 263 & 492 \\
\hline & 10 & Right & 1220 & $6 \mathrm{MV}$ & $6 \mathrm{MV} \mathrm{FFF}$ & 4 & 3 & 274 & 700 \\
\hline \multirow[t]{12}{*}{ Halcyon } & 11 & Left & 1016 & $6 \mathrm{MV}$ & 6MV FFF & 4 & 2 & 277 & 536 \\
\hline & 12 & Left & 798 & $6 \mathrm{MV}$ & 6MV FFF & 4 & 2 & 259 & 415 \\
\hline & 13 & Left & 589 & $6 \mathrm{MV}$ & $6 \mathrm{MV} F F F$ & 4 & 2 & 266 & 575 \\
\hline & 14 & Left & 861 & $6 \mathrm{MV}$ & $6 \mathrm{MVFFF}$ & 4 & 2 & 254 & 484 \\
\hline & 15 & Left & 1035 & $6 \mathrm{MV}$ & $6 \mathrm{MV} F F F$ & 4 & 2 & 254 & 641 \\
\hline & 16 & Right & 1439 & $6 \mathrm{MV}$ & 6MV FFF & 4 & 2 & 266 & 536 \\
\hline & 17 & Right & 494 & $6 \mathrm{MV}$ & $6 \mathrm{MV} F F F$ & 4 & 2 & 243 & 459 \\
\hline & 18 & Right & 496 & $6 \mathrm{MV}$ & $6 \mathrm{MV}$ FFF & 4 & 2 & 246 & 596 \\
\hline & 19 & Right & 879 & $6 \mathrm{MV}$ & $6 \mathrm{MV} F F F$ & 5 & 2 & 274 & 495 \\
\hline & 20 & Right & 792 & $6 \mathrm{MV}$ & 6MV FFF & 4 & 2 & 251 & 618 \\
\hline & Mean & & 813.5 & & & 4.2 & 2.1 & 259.8 & 515.2 \\
\hline & SD & & 283.5 & & & 0.4 & 0.2 & 10.1 & 75.6 \\
\hline
\end{tabular}


of the Elekta Synergy Agility plans. A 3D-CRT field in field technique was used with flattened $6 \mathrm{MV}$ and 10 MV beams in forward planning mode [4]. The gantry angle was optimized in the beam's eye view (BEV) for a minimum lung area and beam divergence toward the lung was compensated by adjusting the gantry angles of the beams accordingly. The beams exceeded the patient surface by minimum of $2 \mathrm{~cm}$. One to two segments per field were added to improve target coverage and dose homogeneity and to omit wedges. This procedure of treatment planning for the Synergy platform resulted in two tangential beams and one to three additional segments with the same tangential gantry angles (4 5 beams in total, see Table 1). Final dose was calculated using the collapsed cone algorithm and a grid size of $0.3 \mathrm{~cm}$.

Treatment planning for the Halcyon system was performed in Eclipse (Varian Medical Systems, Palo Alto, CA, USA, version 15.6). Tangential fields were defined using $6 \mathrm{MV}$ FFF beams. The dose distribution was optimized with a sliding window technique (IMRT). A virtual bolus was added on top of the PTV during the optimization process with a density of $0.26 \mathrm{~g} / \mathrm{cm}^{3}$ and a thickness of $2 \mathrm{~cm}$. With a minimum dose objective for the virtual bolus the optimizer was forced to enlarge the field size into the air. An uncertainty analysis was carried out by shifting the isocenter $(1 \mathrm{~cm})$ in dorsal and contralateral direction which should result in comparable target coverage for PTV-5 mm. For final dose calculation, the bolus was removed. No bolus was used during treatment. The same tangential beam arrangement was used for the Halcyon and Synergy Agility plans. For Halcyon, no additional segment fields were needed due to the sliding window technique which resulted in 2-3 beams (see Table 1). No couch rotation was utilized in the clinical plans for any of the patients selected. In Eclipse, dose was calculated with a grid size of $0.25 \mathrm{~cm}$ using the Acuros External Beam Algorithm, version 15.6.06.

\section{Treatment plan comparison}

We compared the dose-volume histograms (DVHs) of the Halcyon with the Synergy Agility plans. DVH parameters are listed in Table 2. For PTV, the dose received by $2 \%, 50 \%, 80 \%, 95 \%$ and $98 \%$ volume (D02, D 50, D 80 , D95, D98) were evaluated. The homogeneity index (HI) was defined as $\mathrm{HI}=(\mathrm{D} 02-\mathrm{D} 98) / \mathrm{D} 50$. For OARs, the maximum and mean doses were assessed. The number of monitor units (MUs) and the correlation between PTV size and number of MU were analysed for all plans. Both machines (Synergy Agility and Varian Halcyon) were calibrated equally: a dose of $1 \mathrm{~Gy}$ in $10 \mathrm{~cm}$ depth corresponds to $100 \mathrm{MU}$.
Table 2 DVH comparison between Synergy and Halcyon plans

\begin{tabular}{lccl}
\hline & Synergy Agility & Halcyon & p value \\
\hline PTV & & & \\
D95 in Gy & $33.9 \pm 2.3$ & $33.5 \pm 2.5$ & NS* \\
D80 in Gy & $38.0 \pm 0.5$ & $38.6 \pm 0.4$ & $p<0.01$ \\
$D_{\text {Max }}$ in Gy & $43.0 \pm 0.6$ & $43.3 \pm 0.8$ & NS \\
HI & $0.13 \pm 0.01$ & $0.12 \pm 0.02$ & NS \\
PTV-0.5 & & & \\
$D_{\text {Min in Gy }}$ & $35.3 \pm 1.0$ & $35.4 \pm 0.2$ & NS \\
D95 in Gy & $38.5 \pm 0.2$ & $38.8 \pm 0.3$ & 0.03 \\
$D_{\text {Mean in Gy }}$ & $40.1 \pm 0.1$ & $40.1 \pm 0.1$ & NS \\
SD in Gy & $1.0 \pm 0.1$ & $0.8 \pm 0.1$ & 0.01 \\
Lung ipsilateral & & & \\
$D_{\text {Mean in Gy }}$ & $4.7 \pm 0.9$ & $4.4 \pm 1.1$ & NS \\
Lung contralateral & & & \\
$D_{\text {Mean }}$ in Gy & $0.3 \pm 0.1$ & $0.2 \pm 0.1$ & $p<0.01$ \\
Breast contralateral & & & \\
$D_{\text {Mean }}$ in Gy & $0.5 \pm 0.2$ & $0.3 \pm 0.1$ & $p<0.01$ \\
D01 in Gy & $1.8 \pm 0.7$ & $1.0 \pm 0.5$ & $p<0.01$ \\
Heart & & & \\
$D_{\text {Max }}$ in Gy & $31.7 \pm 10.5$ & $33.0 \pm 8.4$ & $\mathrm{NS}$ \\
$D_{\text {Mean }}$ in Gy & $1.7 \pm 0.4$ & $1.5 \pm 0.5$ & $p<0.01$ \\
\hline Dose to trget & & &
\end{tabular}

Dose to target volumes and organs at risk in Synergy Agility plans and Halcyon plans. Stated values indicate mean \pm SD and statistical analysis was performed using the Wilcoxon test. The differences were considered statistically significant when $p<0.05$

${ }^{*}$ Not significant (NS)

** Only left-sided plans were considered for the evaluation of the heart dose

\section{Dosimetric evaluation \\ Plan specific $Q A$}

In order to test the deliverability of the Halcyon treatment plans, plan specific QA was performed. Plans were delivered to an ArcCHECK Phantom (Sun Nuclear Corporation, Melbourne, FL, USA) with the cavity plug present equipped with a Semiflex 31,010 ionization chamber (PTW-Freiburg, Freiburg, Germany) in the center. For point dose measurement, passing criteria were percent dose deviation of less than $3 \%$ from the expected dose. The ArcCHECK measurements were evaluated with $3 \% / 2 \mathrm{~mm}$ and $2 \% / 2 \mathrm{~mm}$ gamma criteria with tolerance level of $96.5 \%$ and $95 \%$ of pixel passing, global normalization and $10 \%$ low dose threshold. Portal dosimetry was evaluated for each field with a gammy criterion of $2 \% / 1 \mathrm{~mm}$ and a tolerable passing rate of $94 \%$.

\section{Imaging and treatment dose}

To evaluate the imaging and treatment dose a left-sided treatment plan was generated for a dynamic thorax phantom (CIRS Incorporated, Norfolk, Virginia, USA). The phantom was used in static mode. Breast surrogates were 
attached to simulate a breast treatment. Breast surrogates were created from silicon material with 3 inserts for an ionization chamber for each side (see Fig. 1). A Unidos dosimeter (PTW-Freiburg, Freiburg, Germany) with $0.3 \mathrm{~cm}^{3}$ thimble chamber (type TM31013) was used for dose measurements. The dosimetric influence of different imaging modes (MV planar imaging, volumetric $\mathrm{kV}$ $\mathrm{CBCT}$ ) was compared by measuring the dose in the ipsilateral and contralateral breast. In total, six point dose values were acquired for each imaging setup. For the Halcyon CBCT system, the breast mode with $125 \mathrm{kV}$ and $45 \mathrm{mAs}$ was used followed by iterative image reconstruction. This reconstruction algorithm is designed to reduce noise and to enhance image quality with high resolution. MV portal imaging is limited to $0^{\circ}$ and $90^{\circ}$ gantry position for the Halcyon system. For the Synergy Agility treatment setup, imaging dose of $\mathrm{kV}$ CBCT $(120 \mathrm{kV}$, $70.4 \mathrm{mAs}, \mathrm{S} 20)$ was compared with $\mathrm{MV}$ images at $0^{\circ}$ gantry position followed by the tangential treatment angles.

For this anthropomorphic breast phantom, treatment plans were optimized for both the Synergy Agility and Halcyon machines. For our standard protocol, imaging and treatment doses were measured and the accumulated dose in the contralateral breast was determined. A typical imaging procedure on the Synergy Agility system would be to perform imaging with anterior-posterior and tangential MV portals before the first treatment followed by imaging once a week (3 fractions of MV imaging and 15 treatment fractions). In contrast, the treatment on the Halcyon system required imaging before each treatment fraction (15 CBCT imaging fractions and 15 treatment fractions).

\section{Statistical analysis}

A statistical comparison of the Halcyon and the Synergy Agility plans was implemented to analyze dosimetric differences between the two machines using the Wilcoxon test for bound comparisons. The differences were

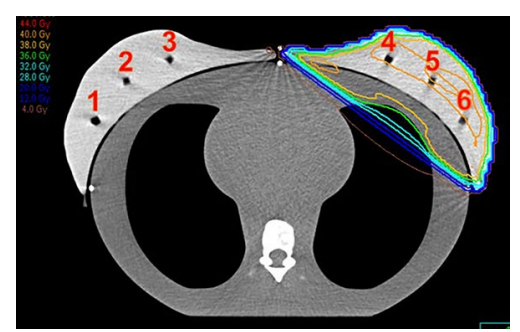

Fig. 1 CIRS phantom with breast surrogates. Measurement of imaging and treatment dose was performed using a CIRS phantom with breast surrogates. Point dose was measured for the contralateral $(1-3)$ and ipsilateral side (4-6). The treatment plan for the Synergy Agility is illustrated considered statistically significant when $p<0.05$. IBM SPSS Statistics for Windows, version 26 (IBM Corp., Armonk, N.Y., USA) was used for statistical evaluation.

\section{Results}

\section{Treatment plan comparison}

The beam configuration for Halcyon and Synergy Agility plans are listed in Table 1. For most cases, $6 \mathrm{MV}$ and 4 fields were sufficient for the field-in-field technique with the Synergy Agility. In 3 out of 20 cases, a field arrangement of 5 beams was needed. A mixture of $6 \mathrm{MV}$ and $10 \mathrm{MV}$ was used in 3 cases. For treatment planning on the Halcyon unit, two treatment fields were used for application of a sliding window technique. Just for one case 3 fields were applied.

Table 1 outlines the difference in MUs for the Halcyon vs. Synergy Agility plans. The mean number of MUs used for the Halcyon delivered plans was 515 MUs, whereas the Synergy Agility delivered plans used 260 MUs $(p<0.001)$. No significant correlation was found between PTV size and number of MUs $\left(R^{2}=0.5\right.$ and $R^{2}=0.1$ for Halcyon and Synergy Agility plans, respectively).

Overall, the plan quality of Halcyon plans was comparable to plans delivered on Elekta Synergy linac. Mean dose values in the PTV and OARs are listed in Table 2. For both treatment units the homogeneity index (HI) was similar: Synergy Agility plans achieved HI of 0.13 while the Halcyon plans achieved 0.12. Slightly improved dose homogeneity was observed for the Halcyon plans. Only the D80 in PTV differed significantly by 0.6 Gy on average $(p<0.01)$. The SD in PTV-5 mm was $1.0 \mathrm{~Gy}$ and 0.8 Gy on average for Synergy Agility and Halcyon plans, respectively. Significant differences were observed for D95 and SD in PTV-5 mm. However, given that dose differences are very low, we do not consider them of clinical relevance. Nominal target coverage (D95 and D80) was comparable for Halcyon and Synergy Agility plans.

Mean dose in the lungs and heart was slightly improved for Halcyon plans. Significant differences were found for the mean dose in the contralateral lung $(0.1 \mathrm{~Gy})$, the heart $(0.2 \mathrm{~Gy})$ and the contralateral breast (0.2). Maximum dose in the heart was 1.3 Gy lower on average for Synergy Agility plans but not statistically significant.

\section{Plan specific QA}

Phantom measurements with the ArcCHECK and portal dosimetry were performed for the Halcyon plans. All plans were successfully delivered on the Halcyon unit. Table 3 shows the ion chamber measurements and gamma passing rates for all patient plans. For the ArcCHECK, the mean deviation of the ion chamber dose was $-1 \%$ with a maximum deviation of $-2.7 \%$. The pass rate 
Table 3 Plan specific QA results

\begin{tabular}{|c|c|c|c|c|c|}
\hline \multirow[t]{2}{*}{ Patient } & \multirow{2}{*}{$\begin{array}{l}\text { lon chamber dose } \\
\text { deviation in } \%\end{array}$} & \multicolumn{2}{|l|}{ Portal dosimetry } & \multicolumn{2}{|l|}{ ArcCHECK } \\
\hline & & $\begin{array}{l}\text { Field \#1 } \\
2 \% / 1 \mathrm{~mm}>94 \%\end{array}$ & $\begin{array}{l}\text { Field \#2 } \\
2 \% / 1 \mathrm{~mm}>94 \%\end{array}$ & $3 \% / 2 \mathrm{~mm}>96,5 \%$ & $2 \% / 2 \mathrm{~mm}>95 \%$ \\
\hline 1 & -2.7 & 99.3 & 96.6 & 100 & 100 \\
\hline 2 & -1.6 & 99.5 & 96.2 & 100 & 98.3 \\
\hline 3 & -1.6 & 99.3 & 96 & 100 & 98.6 \\
\hline 4 & -1.5 & 99.5 & 97.1 & 99.8 & 97.2 \\
\hline 5 & -1.9 & 99.3 & 96.5 & 99.7 & 96.9 \\
\hline 6 & -1.3 & 96.8 & 98 & 100 & 98.8 \\
\hline 7 & -1.2 & 95.3 & 97.6 & 99.7 & 96.8 \\
\hline 8 & -1.0 & 97.7 & 98.1 & 100 & 99.1 \\
\hline 9 & -1.3 & 96.0 & 97.5 & 100 & 99.6 \\
\hline 10 & -1.3 & 96.7 & 97.2 & 98.4 & 92.2 \\
\hline 11 & -1.2 & 98.4 & 97.5 & 97.6 & 95.5 \\
\hline 12 & -1.2 & 99.5 & 97.3 & 99.5 & 95.1 \\
\hline 13 & -1.0 & 98.9 & 97.4 & 100 & 98.9 \\
\hline 14 & -0.8 & 98.9 & 96.6 & 99.8 & 96.5 \\
\hline 15 & -1.1 & 99.2 & 94.6 & 98.9 & 92 \\
\hline 16 & -1.6 & 97.8 & 96 & 97.6 & 88.3 \\
\hline 17 & -1.6 & 94.8 & 97.2 & 99.7 & 97.8 \\
\hline 18 & 2.5 & 94.6 & 95.8 & 98.5 & 95.8 \\
\hline 19 & 2.4 & 97.1 & 97.5 & 99.8 & 99 \\
\hline 20 & -0.5 & 97.2 & 95.8 & 100 & 99.2 \\
\hline Mean & -1.0 & 97.8 & 96.7 & 99.5 & 96.8 \\
\hline SD & 1.3 & 1.7 & 0.9 & 0.8 & 3.0 \\
\hline
\end{tabular}

Results of the plan specific QA for ion chamber measurement, portal dosimetry and the ArcCHECK phantom

of the diode measurements was $99.5 \%$ for the $3 \% / 2 \mathrm{~mm}$ gamma criterion.

All portal dosimetry measurements passed the $3 \% / 2 \mathrm{~mm}$ gamma criterion with $100 \%$ of the pixels. The evaluation of the $2 \% / 1 \mathrm{~mm}$ gamma criterion resulted in a mean pass rate of $97.8 \%$ and $96.7 \%$ for field $\# 1$ and field $\# 2$, respectively.

\section{Imaging and treatment dose}

For treatment setup on a Synergy Agility unit, the imaging dose of MV portals was compared with $\mathrm{kV}$ CBCT. A summary of all measurements is given in Table 4 and Fig. 2. The lowest imaging dose in the contralateral breast (points 1-3) was measured for the anterior-posterior and tangential MV portals on the Synergy Agility (in total $6 \mathrm{MU}$ ). Dose in points 1,2 and 3 was $0.1 \mathrm{mGy}$, $0.1 \mathrm{mGy}$, and $0.4 \mathrm{mGy}$, respectively. Slightly larger doses were measured for the $\mathrm{kV} \mathrm{CBCT}$ setup with $0.1 \mathrm{mGy}$, $0.2 \mathrm{mGy}$ and $0.8 \mathrm{mGy}$ in points $1-3$. The cumulated dose of all treatment and imaging fractions for the Synergy Agility plan would result in $195.2 \mathrm{mGy}, 291.8 \mathrm{mGy}$ and $541.1 \mathrm{mGy}$ in points 1,2 and 3 of the contralateral breast, respectively (Table 4). Dose was accumulated for 3 fractions of MV imaging and 15 treatment fractions.

For treatment setup on the Halcyon unit, the imaging dose of MV portals was compared with the kV CBCT. The MV portals deliver more dose to the contralateral breast than the $\mathrm{kV} \mathrm{CBCT}$. For MV portal imaging from $0^{\circ}$ and $90^{\circ}$ gantry angle, dose measurement in points $1-3$ resulted in $3.5 \mathrm{mGy}, 3.2 \mathrm{mGy}$ and $3.8 \mathrm{mGy}$ respectively. The imaging dose of the Halcyon $\mathrm{kV} \mathrm{CBCT}$ is comparable with the Synergy Agility CBCT. Dose values in points 1-3 was $1.0 \mathrm{mGy}, 0.6 \mathrm{mGy}$ and $1.0 \mathrm{mGy}$ respectively. For treatment on the Halcyon unit setup imaging must be performed daily. The cumulated dose of all treatment and imaging fractions for the Halcyon plan would result in lower doses with $102.3 \mathrm{mGy}, 141.1 \mathrm{mGy}$ and $261.9 \mathrm{mGy}$ in points 1,2 and 3 of the contralateral breast, respectively (Table 4). In this case, dose was accumulated for 15 fractions of CBCT imaging and 15 treatment fractions. Thus, our standard approach with weekly MV imaging at the Synergy linac resulted in a higher dose for the contralateral breast than the daily $\mathrm{kV}$ imaging at the Halcyon linac. 
Table 4 Imaging and treatment dose

\begin{tabular}{|c|c|c|c|c|c|c|c|}
\hline & & \multicolumn{6}{|c|}{ Dose in mGy } \\
\hline & & \multicolumn{3}{|c|}{ Contralateral side } & \multicolumn{3}{|c|}{ Ipsilateral side } \\
\hline & & 1 & 2 & 3 & 4 & 5 & 6 \\
\hline \multirow[t]{6}{*}{ Synergy Agility } & $\mathrm{kV} C B C T$ & 0.1 & 0.2 & 0.8 & 1.9 & 2.0 & 2.9 \\
\hline & MV portals* & 0.1 & 0.1 & 0.4 & 52.5 & 46.5 & 39.7 \\
\hline & Treatment & 13.0 & 19.4 & 36.0 & 2737.1 & 2711.8 & 2704.2 \\
\hline & 15 fx treatment & 195.0 & 291.3 & 540.0 & $41,056.5$ & $40,677.2$ & $40,563.4$ \\
\hline & 3 fx imaging & 0.2 & 0.4 & 1.1 & 157.5 & 139.4 & 119.1 \\
\hline & Total & 195.2 & 291.8 & 541.1 & $41,213.9$ & $40,816.6$ & $40,682.5$ \\
\hline \multirow[t]{6}{*}{ Halcyon } & $\mathrm{kV} C B C T^{*}$ & 1.0 & 0.6 & 1.0 & 2.6 & 2.6 & 2.6 \\
\hline & MV portals & 3.5 & 3.2 & 3.8 & 23.9 & 25.8 & 24.5 \\
\hline & Treatment & 5.9 & 8.8 & 16.5 & 2649.2 & 2634.7 & 2645.0 \\
\hline & $15 \mathrm{fx}$ treatment & 88.0 & 131.6 & 247.5 & $39,737.7$ & $39,519.8$ & $39,675.4$ \\
\hline & 15 fx imaging & 14.3 & 9.6 & 14.3 & 38.3 & 38.3 & 38.3 \\
\hline & Total & 102.3 & 141.1 & 261.9 & $39,776.0$ & $39,558.0$ & $39,713.7$ \\
\hline
\end{tabular}

Dose was measured for the ipsilateral and contralateral side in the CIRS phantom for $\mathrm{kV}$ and MV imaging procedures and one treatment fraction. Total dose for our standard imaging protocols (marked with *) and all treatment fractions is listed

\section{Discussion}

The presented work shows that the Halcyon linac is suitable for whole-breast irradiation using a tangential beam technique. A practical and efficient planning method for delivering 3D conformal breast radiotherapy using the Halcyon was implemented in our department. The simplified workflow on the Halcyon system enables a fast delivery with a reduced probability of patient collisions due to the closed ring gantry design and collision warning during treatment planning.

The overall plan quality regarding target volumes and OARs was comparable for plans delivered on Halcyon and Synergy Agility machines. Homogeneity index was 0.13 and 0.12 for Synergy and Halcyon plans, respectively. Similar results for the comparison of plan quality of $\mathrm{C}$-arm and Halcyon plans were reported by other groups $[14,15]$. Flores et al. used the irregular surface compensator technique and compared to the results of the current study reported lower homogeneity indices of 0.09 and 0.08 for $\mathrm{C}$-arm and Halcyon linacs, respectively [14]. The increased number of MUs for delivering the Halcyon plans was also observed by other researchers [14-16]. For the current investigation, the MUs were $50 \%$ higher than the field-in-field breast treatment plans using tangential beams with Synergy Agility. The reason is the sliding window technique in combination with the un-flattened beam for the Halcyon plans.

In the current work, lower mean doses in the OARs were observed for the Halcyon plans, while the maximum heart dose was on average 1.3 Gy higher for the Halcyon plans. In contrast, Flores et al. and Morris et al. observed an increase in the mean dose in the contralateral lung and the heart for the Halcyon plans compared to C-arm linacs $[14,15]$.

Dose to OARs needs to be considered when choosing the setup imaging technique (MV portals or CBCT). Comparable imaging dose for $\mathrm{kV} \mathrm{CBCT} \mathrm{was} \mathrm{observed}$ for both systems in the current work. However, the Halcyon requires $\mathrm{CBCT}$ imaging to be performed at every fraction. Regarding the sum of imaging and treatment dose for our standard protocol, much lower doses to the contralateral breast were determined with the Halcyon unit. This is mainly due to the lower interleaf transmission for the Halcyon MLC and a contribution of scattered dose from the collimator system. Additional low dose is caused by the larger number of beams for the Synergy plans (4-5 beams) compared to Halcyon plans (2-3 beams). The Halcyon MLC has $77 \mathrm{~mm}$ thick leaves in each bank, creating a combined thickness of $154 \mathrm{~mm}$. The combined transmission through the dual layer MLC is less than $0.01 \%$ at $6 \mathrm{MV}$ FFF $[15,17]$. Whereas for the Agility MLC the transmission through the MLC is $0.44 \%$ at $6 \mathrm{MV}$ and $0.52 \%$ at $10 \mathrm{MV}$ [18]. Flores et al. suggested a dose reduction for the OARs by using angled MV portals or non-ionizing techniques like surface guided RT [14].

The plan specific QA indicate a good agreement between the measured and calculated dose. The point dose measurements in the ArcCHECK phantom showed an average and maximum deviation of $-1 \%$ and $-2.7 \%$ respectively. Similar findings were reported by Morris et al. with an average and maximum point dose deviation of $1.5 \%$ and $2.23 \%$ in a solid water phantom [15]. In the current work, the average ArcCHECK gamma passing rate was $99.5 \%$ for $3 \% / 2 \mathrm{~mm}$ criterion. 
(a)

IMAGING DOSE
Contralateral

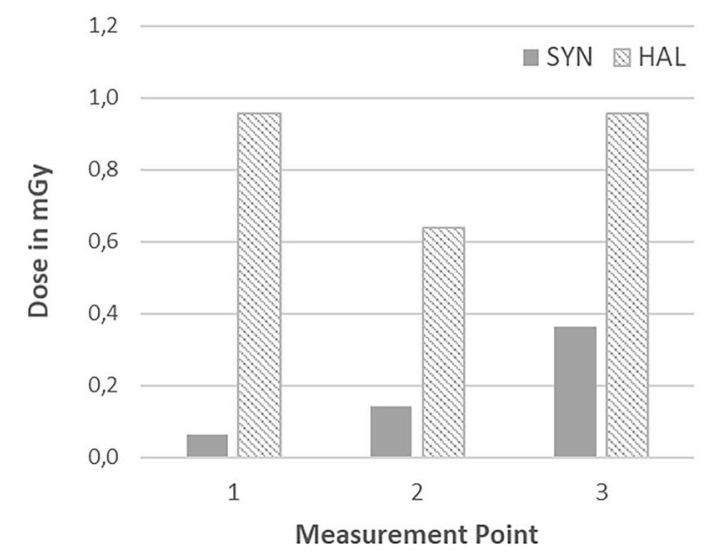

(c)
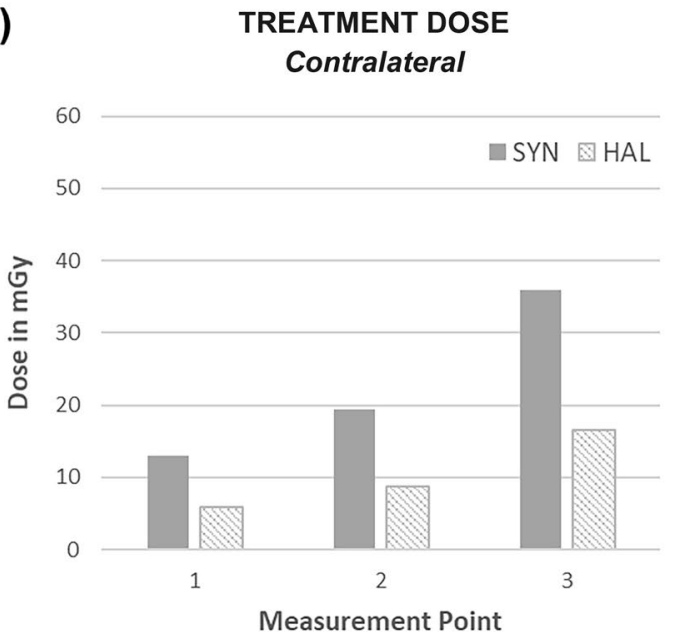

(b)

IMAGING DOSE

Contralateral

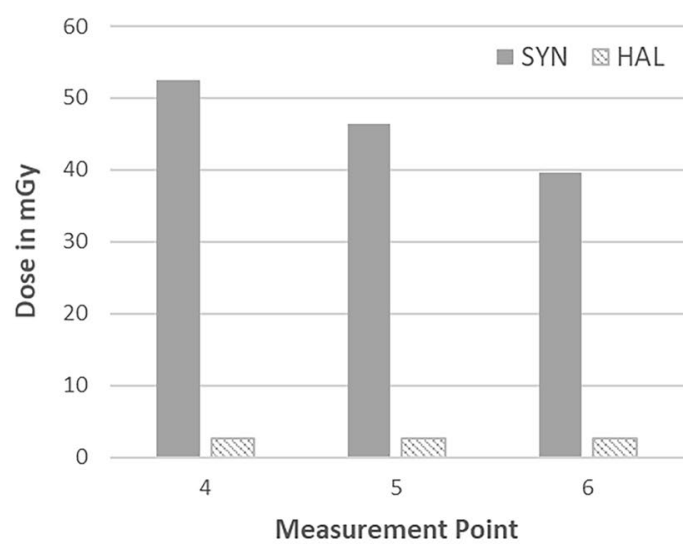

(d) TREATMENT DOSE Ipsilateral

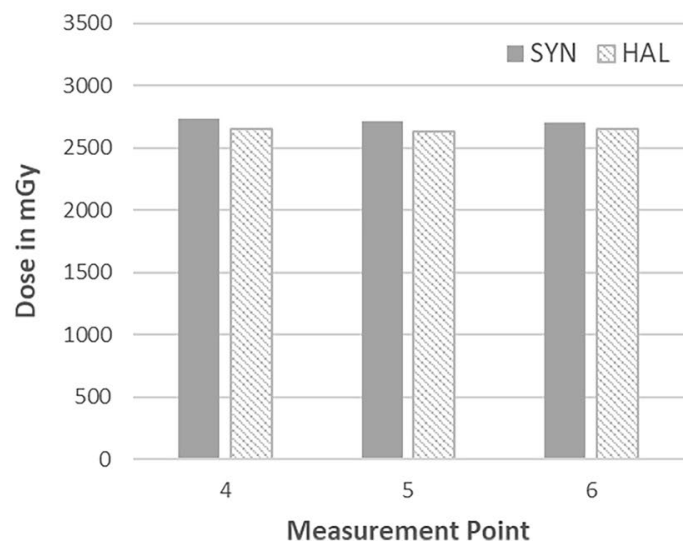

Fig. 2 Imaging and treatment dose. Dose contribution by imaging with thestandard protocol $(\mathbf{a}, \mathbf{b})$ and by one treatment fraction $(\mathbf{c}, \mathbf{d})$ is displayed for the Synergy Agility (grey) and Halcyon unit (striped area). Results for the contralateral breast (points 1-3) are plotted on the left (a, $\mathbf{c}$ ). The results for the ipsilateral breast (points 4-6) are shown at the right (b, d)

Morris et al. published high pass rates of $99.4 \%$ for plan specific QA with Delta4 [15]. Portal dosimetry yielded pass rates of $100 \%$ for the $3 \% / 2 \mathrm{~mm}$ criterion which is consistent with the results published by Morris et al. [15]. In order to get some variation the $2 \% / 1 \mathrm{~mm}$ gamma criterion was used, still resulting in mean gamma pass rates above $96 \%$.

A common technique to spare the heart for left-sided treatments is deep inspiration breath-hold (DIBH). Barsky et al. reported about initial experience in treating patients with breast cancer on a Halcyon system in either supine position, with or without DIBH, or prone position. DIBH was used in $29 \%$ of the patients with average treatment times of $5.8 \mathrm{~min}$ [19]. Kennedy stated the total treatment time can be drastically reduced using irregular surface compensator technique as opposed to the Dynamic Flattening Beam-enabled field-in-field technique [16].

A bolus is commonly applied to improve target coverage near the surface while also enhancing the risk of severe skin reactions and a possible negative impact on cosmesis. The effect on superficial dose for $6 \mathrm{MV}$ FFF beams and the need for bolus was evaluated by O'Grady et al. [20]. An increased superficial dose was observed for the use of a Halcyon 6MV FFF beam compared to $6 \mathrm{MV}$ beams on C-Arm linacs for whole breast irradiation. It was assumed by O' Grady et al. that the increased dose is primarily caused by a higher proportion of low energy photons. The traditional application of a bolus should be carefully re-evaluated for $6 \mathrm{MV}$ beams [20]. 
In the current work, we only investigated tangential beam arrangements. Several modalities are available for whole breast irradiation, e.g., the Irregular Surface Compensation technique and dynamic flatting beam sequences. For target volumes including the mammaria interna lymph nodes, we currently use VMAT and multi field IMRTs (consisting of 13 to 19 beams) on Synergy Platforms. The possibilities of treating such cases with the Halcyon will be investigated in a subsequent examination.

The field size of the Halcyon system is limited to $28 \times 28 \mathrm{~cm}^{2}$. A multi isocenter technique allows an extension up to a field size of $36 \mathrm{~cm}$ in longitudinal direction with a maximum isocenter shift of $8 \mathrm{~cm}$. Patients with a PTV extension of more than $36 \mathrm{~cm}$ are currently not suited for treatment on a Halcyon unit (Version 2.0).

\section{Conclusions}

We implemented a treatment technique with a tangential beam arrangement for whole breast irradiation. Plan quality was equally good on Halcyon and Synergy Agility machines. Monitor units increased on average by $50 \%$ for Halcyon plans due to the sliding window technique. A significantly lower dose to the contralateral breast was measured for the Halcyon plans due to less interleaf transmission of Halcyon's dual layer MLC and some scattered dose from the collimator system.

\begin{abstract}
Abbreviations
3D CRT: Three-dimensional conformal radiotherapy; BEV: Beams eye view; CBCT: Cone beam computer tomography; CTV: Clinical target volume; DIBH: Deep inspiration breath-hold; DVH: Dose volume histogram; FFF: Flattening filter free; HI: Homogeneity index; IGRT: Image guided radiation therapy; IMRT: Intensity modulated radiation therapy; MLC: Multileaf collimator; MU: Monitor unit; OAR: Organ at risk; PTV: Planning target volume; QA: Quality assurance; SD: Standard deviation; VMAT: Volumetric modulated arc therapy.
\end{abstract}

\section{Acknowledgements}

Breast surrogates for the phantom measurements were made by Jonas Ringholz using a 3D printer approach.

\section{Authors' contributions}

AR, SoW, BP, KIB, OS and MF designed the analysis. AR, KaB, GR, SoW, StW and $F E$ participated in the data collection and performed the analysis. All authors performed critical review of the manuscript and finally approved the manuscript.

\section{Funding}

This publication was supported by the Open Access Publication Fund of the University of Wuerzburg.

\section{Availability of data and materials}

Research data are stored in an institutional repository and will be shared upon request to the corresponding author.

\section{Declarations}

\section{Ethics approval and consent to participate}

Ethical review and written informed consent was not required for participation in this retrospective analysis in accordance with the local legislation (BayKrG Art. 27 (4)) and institutional requirements. Written informed consent for treatment was provided by all patients.

\section{Consent for publication}

Not applicable.

\section{Competing interests}

The authors declare that they have no competing interests.

Received: 13 August 2021 Accepted: 28 October 2021

Published online: 06 November 2021

\section{References}

1. Wockel A, Festl J, Stuber T, Brust K, Krockenberger M, Heuschmann PU, Jiru-Hillmann S, Albert US, Budach W, Follmann M et al: Interdisciplinary Screening, Diagnosis, Therapy and Follow-up of Breast Cancer. Guideline of the DGGG and the DKG (S3-Level, AWMF Registry Number 032/045OL, December 2017) - Part 2 with recommendations for the therapy of primary, recurrent and advanced breast cancer. Geburtshilfe Frauenheilkd 2018, 78(11):1056-1088.

2. Corradini S, Reitz D, Pazos M, Schonecker S, Braun M, Harbeck N, Matuschek C, Bolke E, Ganswindt U, Alongi F et al: Mastectomy or breastconserving therapy for early breast cancer in real-life clinical practice: outcome comparison of 7565 cases. Cancers (Basel) 2019, 11(2).

3. Litiere S, Werutsky G, Fentiman IS, Rutgers E, Christiaens MR, Van Limbergen E, Baaijens MH, Bogaerts J, Bartelink H. Breast conserving therapy versus mastectomy for stage I-II breast cancer: 20 year follow-up of the EORTC 10801 phase 3 randomised trial. Lancet Oncol. 2012;13(4):412-9.

4. Ludwig V, Schwab F, Guckenberger M, Krieger T, Flentje M. Comparison of wedge versus segmented techniques in whole breast irradiation: effects on dose exposure outside the treatment volume. Strahlenther Onkol. 2008;184(6):307-12.

5. Stovall M, Smith SA, Langholz BM, Boice JD Jr, Shore RE, Andersson M, Buchholz TA, Capanu M, Bernstein L, Lynch CF, et al. Dose to the contralateral breast from radiotherapy and risk of second primary breast cancer in the WECARE study. Int J Radiat Oncol Biol Phys. 2008;72(4):1021-30.

6. Franco P, Zeverino M, Migliaccio F, Sciacero P, Cante D, Casanova Borca V, Torielli P, Arrichiello C, Girelli G, Numico G, et al. Intensity-modulated adjuvant whole breast radiation delivered with static angle tomotherapy (TomoDirect): a prospective case series. J Cancer Res Clin Oncol. 2013;139(11):1927-36.

7. Hashimoyo H, Omura M, Matsui K, Mukai Y, Hongo H, Yamakabe W, Saito K, Yoshida M. Tangent field technique of TomoDirect improves dose distribution for whole-breast irradiation. J Appl Clin Med Phys. 2015;16(3):5369.

8. Lauche O, Kirova YM, Fenoglietto P, Costa E, Lemanski C, Bourgier C, Riou O, Tiberi D, Campana F, Fourquet A, et al. Helical tomotherapy and volumetric modulated arc therapy: New therapeutic arms in the breast cancer radiotherapy. World J Radiol. 2016;8(8):735-42.

9. Schubert LK, Gondi V, Sengbusch E, Westerly DC, Soisson ET, Paliwal BR, Mackie TR, Mehta MP, Patel RR, Tome WA, et al. Dosimetric comparison of left-sided whole breast irradiation with 3DCRT, forward-planned IMRT, inverse-planned IMRT, helical tomotherapy, and topotherapy. Radiother Oncol. 2011;100(2):241-6.

10. Cozzi L, Fogliata A, Nicolini G, Bernier J. Clinical experience in breast irradiation with intensity modulated photon beams. Acta Oncol. 2005;44(5):467-74.

11. Zhang Q, Yu XL, Hu WG, Chen JY, Wang JZ, Ye JS, Guo XM. Dosimetric comparison for volumetric modulated arc therapy and intensity-modulated radiotherapy on the left-sided chest wall and internal mammary nodes irradiation in treating post-mastectomy breast cancer. Radiol Oncol. 2015;49(1):91-8. 
12. Bratengeier K, Oechsner M, Gainey M, Flentje M. Remarks on reporting and recording consistent with the ICRU reference dose. Radiat Oncol. 2009;4:44.

13. Offersen BV, Boersma LJ, Kirkove C, Hol S, Aznar MC, Sola AB, Kirova YM, Pignol JP, Remouchamps V, Verhoeven Ket al: ESTRO consensus guideline on target volume delineation for elective radiation therapy of early stage breast cancer, version 1.1. Radiother Oncol 2016, 118(1):205-208.

14. Flores-Martinez E, Kim GY, Yashar CM, Cervino LI. Dosimetric study of the plan quality and dose to organs at risk on tangential breast treatments using the Halcyon linac. J Appl Clin Med Phys. 2019;20(7):58-67.

15. Morris R, Laugeman E, Hilliard J, Zoberi I, Heerman A, Hugo G, Mutic S, Cai B. Field-in-field breast planning for a jawless, double-stack MLC LINAC using flattening-filter-free beams. J Appl Clin Med Phys. 2019;20(11):14-26.

16. Kennedy C, Freedman G, Taunk N, Scheuermann R, Dong L, Metz JM, Li $\mathrm{T}$ : Whole breast irradiation with Halcyon 2.0: workflow and efficiency of field-in-field treatment with dynamic beam flattening technique and kv cone beam computed tomography. Cureus 2018, 10(10):e3510.

17. Kim MM, Bollinger D, Kennedy C, Zou W, Scheuermann R, Teo BK, Metz JM, Dong L, LiT. Dosimetric characterization of the dual layer
MLC system for an O-ring linear accelerator. Technol Cancer Res Treat. 2019;18:1533033819883641.

18. Thompson CM, Weston SJ, Cosgrove VC, Thwaites DI: A dosimetric characterization of a novel linear accelerator collimator. Med Phys 2014, 41(3):031713.

19. Barsky AR, O'Grady F, Kennedy C, Taunk NK, Dong L, Metz JM, Li T, Freedman GM. Initial clinical experience treating patients with breast cancer on a 6-MV flattening-filter-free O-ring linear accelerator. Adv Radiat Oncol. 2019;4(4):571-8.

20. O'Grady F, Barsky AR, Anamalayil S, Freedman GM, Kennedy C, Cai B, Laugeman E, Dong L, Hugo GD, Metz JM, et al. Increase in superficial dose in whole-breast irradiation with halcyon straight-through linac compared with traditional c-arm linac with flattening filter: in vivo dosimetry and planning study. Adv Radiat Oncol. 2020;5(1):120-6.

\section{Publisher's Note}

Springer Nature remains neutral with regard to jurisdictional claims in published maps and institutional affiliations.
Ready to submit your research? Choose BMC and benefit from:

- fast, convenient online submission

- thorough peer review by experienced researchers in your field

- rapid publication on acceptance

- support for research data, including large and complex data types

- gold Open Access which fosters wider collaboration and increased citations

- maximum visibility for your research: over 100M website views per year

At BMC, research is always in progress.

Learn more biomedcentral.com/submissions 